\title{
Adaptive Decomposition Into Mono-Components *
}

\author{
Tao Qian ${ }^{\dagger}$, Yan-Bo Wang ${ }^{\ddagger}$ and Pei Dang ${ }^{\S}$
}

\begin{abstract}
The paper reviews some recent progress on adaptive signal decomposition into mono-components that are defined to be the signals of non-negative analytic phase derivatives.

Key Words: Hilbert transform, analytic signal, analytic phase derivative, mono-component, Bedrosian identity, adaptive decomposition, starlike function, p-starlike function, inner and outer function
\end{abstract}

\section{Introduction}

In 1998, Huang et al ([7]) developed an engineering algorithm to represent signal data, called "empirical mode decomposition" (EMD), with which a complicated data can be decomposed into a sum of finite number, and often a small number of "intrinsic mode functions" (IMFs). Since the decomposition is based on the local characters of the data, it is, in particular, applicable to nonlinear and non-stationary processes.

Instantaneous amplitude, phase and frequency are essential concepts in signal processing and time-frequency analysis for non-linear and non-stationary signals. These concepts are defined via analytic signal. The associated analytic signal $\mathcal{A} f(t)$ of a real signal $f(t) \in L^{p}(\mathbf{R}), 1 \leq p \leq \infty$, is defined by $\mathcal{A} f(t)=f(t)+i \mathcal{H} f(t)$, where $\mathcal{H} f(t)$ is the Hilbert transform of $f(t)$ defined by the principal value integral

$$
\mathcal{H} f(t)=p \cdot v \cdot \frac{1}{\pi} \int_{-\infty}^{\infty} \frac{f(s)}{t-s} d s .
$$

As a counterpart, for periodic functions $f(t) \in L^{p}[0,2 \pi], 1 \leq p \leq \infty$, the associated analytic signal is defined by $\tilde{\mathcal{A}} f(t)=f(t)+i \tilde{\mathcal{H}} f(t)$ where $\tilde{\mathcal{H}} f(t)$ is the

\footnotetext{
* Supported by University of Macau under research grant RG056/01-02S/QT/FST

$\dagger$ Department of Mathematics, Faculty of Science and Technology, University of Macau, Taipa, Macao, China. E-mail:fsttq@umac.mo

${ }^{\ddagger}$ Department of Mathematics, Faculty of Science and Technology, University of Macau, Taipa, Macao, China. E-mail:ya77405@umac.mo

$\S$ Department of Mathematics, Faculty of Science and Technology, University of Macau, Taipa, Macao, China. E-mail:ya77408@umac.mo
} 
Circular Hilbert transform

$$
\tilde{\mathcal{H}} f(t)=p \cdot v \cdot \frac{1}{2 \pi} \int_{-\pi}^{\pi} \cot \left(\frac{t-s}{2}\right) f(s) d s
$$

The Fourier transform of $\mathcal{A} f(t)$ vanishes at negative frequencies. The analytic signal $\mathcal{A} f(t)$ can be written in the polar form $\mathcal{A} f(t)=a(t) e^{i \theta(t)}$ where $a(t)$ is the amplitude and $\theta(t)$ the phase. The phase derivative $\omega(t)=\frac{d \theta(t)}{d t}$ is usually called the instantaneous frequency. This definition for instantaneous frequency, however, causes a lot of controversies. In fact, many do not agree with it, for, from the physics point of view, a quatity must firstly be non-negative and then may be called "frequency". Unfortunately, instantaneous frequency such defined is not always non-negative. The simplest counter examples include the mappings $F\left(e^{i t}\right)=1+p e^{i t}$, where $-1<p<1$. For a fixed such $p$ the mapping $F\left(e^{i t}\right)$ is the analytic signal of the real-valued signal $f\left(e^{i t}\right)=1+p \cos t$ whose boundary phase derivative is a continuous function larger than zero and less than zero, respectively, in adjacent intervals for the $t$-variable. To see this we note that the conformal mapping $F(z)$ maps the unit disc centered at the origin onto a disc centered at 1 that does not contain the origin. Thus the phase function on the boundary is not monotone. For signals like this we should not regard the phase derivative as a well defined "instantaneous frequency". The concerned concept is physically meaningful only when the analytic phase derivative is non-negative almost everywhere.

It was expected ([7]) that IMFs resulted from EMD would have well defined instantaneous frequency functions, or, in other words, non-negative analytic phase derivatives. In 2006, Sharpley and Vatchev constructed examples to show that there exist IMFs whose analytic phase functions are not monotone ([9]). As consequence, physically meaningful analytic instantaneous frequency cannot be defined for general. Practically an IMF is associated with a certain EMD determined by the type of envelops and the thresholds that are in use: If a basic function cannot be further decomposed by the EMD, then it is called an IMF for that EMD. An IMF may not remain to be an IMF any more if, for instance, the threshold changes.

Motivated by Huang et al's algorithm EMD and the related decompositions there have been studies in relation to what we call by mono-components (see below) and the related adaptive decompositions. The present paper is a survey on the main results in this later trend of study but not on the original EMD and its related decompositions. Comparatively, EMD algorithms have very best localization properties but the resulted basic signals, IMFs, are lack of global property: Being of a non-negative analytic instantaneous frequency function is a global property related to conformal mappings. Our approach, being of the global property, does not have promising local properties, and in particular, like Fourier series, is of Gibbs phenomenon. This is no wonder, as the theory of mono-components stretches its roots into analytic function theory. The described situation is as what is stated in the traditional Chinese philosophy: One cannot get fish and bear's paw at the same time (Mencius). 
We make what is expected a mathematical object called mono-component defined as follows.

Definition If real-valued $f=\rho \cos \theta \in L^{p}(R), 1 \leq p \leq \infty$, where $\rho, \theta$ are measurable functions with the properties

$$
\begin{aligned}
H(\rho \cos \theta) & =\rho \sin \theta \\
\rho & \geq 0 \\
\theta^{\prime} & \geq 0
\end{aligned}
$$

then $f$ is called a real mono-component $(M C)([15])$.

One can similarly defines complex mono-component on the line and real (complex)-mono-components on the unit circle ([15]). In the future, we call all the different types of mono-components just mono-components. This simplification does not cause any confusion. Instead of the unrealistic desire that every analytic signal would have instantaneous frequency we seek for decomposition of signals into the basic pieces of well defined instantaneous frequency, viz, the mono-components.

\section{Bedrosian Identity}

The classical Bedrosian theorem is established in 1963 ([1]).

Theorem 2.1. Assume that $f(t)$ and $g(t)$ are complex-valued signals of finite energy and $\mathcal{F}$ and $\mathcal{G}$ are, respectively, their Fourier transforms. If (i) $\mathcal{F}(\omega)=0$ for $|\omega|>\alpha$ and $\mathcal{G}(\omega)=0$ for $|\omega|<\beta$, where $\beta \geq \alpha \geq 0$; or (ii) $\mathcal{F}(\omega)=0$ for $\omega<-\alpha$ and $\mathcal{G}(\omega)=0$ for $\omega<\beta$, where $\beta \geq \alpha>0$, then $\mathcal{H}(f g)(t)=-i f(t) g(t)=f(t) \mathcal{H} g(t)$.

Bedrosian identity may simplify computations of Hilbert transforms of products of functions. In practice, the amplitude-frequency representation of a signal often satisfies the assumptions of the Bedrosian theorem. In fact, amplitude is usually of low-frequencies. The recent study of Bedrosian identity is motivated by the purpose of constructing non-unimodular mono-component.

Below, we briefly list the main results in this study.

Yuesheng Xu and Dunyan Yan in ([21]) investigate a necessary and sufficient condition for Bedrosian identity. The classical Bedrosian theorem is a particular case of the sufficient part of the theorem. If restricted to real signals, use of the theorem, however, is reduced to the case delt with in the classical Bedrosian theorem.

Bo Yu and Haizhang Zhang in ([22]) characterize a class of functions satisfying the classical Bedrosian identity or the circular Bedrosian identity by certain homogeneous semi-convolution equations. The structure of solutions of these equations is then studied by using translation invariant subspaces of Hardy spaces and additive positive definite kernels. The results obtained provide some insights into the Bedrosian identity and are used to construct what they call "intrinsic mode functions". 
Tao Qian, Yuesheng Xu, Dunyan Yan, Lixin Yan, and Bo Yu ([14]) characterize in terms of Fourier spectrum the boundary values of functions in the complex Hardy spaces $H^{p}\left(\mathbb{C}^{ \pm}\right), 1 \leq p \leq \infty$. As application they extend the Bedrosian identity, originally stated for square-integrable functions, to the $L^{p}$ and $L^{q}$ cases. Several sufficient conditions for $f \in L^{p}$ and $g \in L^{q}$ giving rise to the Bedrosian identity are given.

Through a different approach based on the characterization of real Hardy spaces in recent harmonic analysis studies, Lihua Yang and Haizhang Zhang ([23]) also proved the Bedrosian identity for $1 \leq p, q \leq \infty$. They establish a necessary and sufficient condition for $f \in H^{p}$ and $g \in H^{q}$ with $p^{-1}+q^{-1} \leq 1$ to satisfy the Bedrosian identity. As application, they give a characterization of $f$ to satisfy the identity when $g$ is a finite linear combination of complex sinusoids. They also show that if $f$ is of low Fourier frequencies then it is necessary for $g$ to have high Fourier frequencies in order to satisfy the Bedrosian identity.

Lately, Lihui Tan et al ([19], [20]) studied Bedrosian identity by using complex analysis methods, especially the Nevanlinna factorization theorem and the backward shifting operator. While all the previous studies are based on Fourier transformation, their different methodology provides more insightful information and contributes new understanding to the question.

\section{Unimodular cases}

The Bedrosian identity method to produce new mono-component is based on the unimodular cases. By definition ([8]) phase signals are real signals $f(t)=$ $a \cos \theta(t)$, where $a$ is a constant and $e^{i \theta(t)}$ is an analytic signal. For such signals all the information is contained in the phase function. The condition that $e^{i \theta(t)}$ is an analytic signal requires special properties on $\theta(t)$. To the authors' knowledge, Picinbono would be the first person who systematically studied finite Blaschke products as mono-components on the line ([8]).

Tao Qian, Qiuhui Chen, Luoqing Li $([11],[12])$ systematically studied periodic analytic phase signals on the line.

The simplest and nontrivial case is Möbius function. Let $a$ be a complex number in the unit disc $D$, and $\tau_{a}(z)=\frac{z-a}{1-\bar{a} z}$ be the corresponding Möbius transform. Then the unimodular function $\tau_{a}\left(e^{i t}\right), t \in[0,2 \pi]$, is a unimodular analytic signal, and, moreover, a mono-compent. Note that $\tau_{a}$ conformally maps the unit disc to the unit disc, and the unit circle to the unit circle, and keeps the anti-clockwise rotation. Thus the boundary function $\tau_{a}\left(e^{i t}\right)$ is a monocomponent.

This result can be extended to finite or infinite Blaschke products. A finite Blaschke product is a function $B_{n}(z)=\prod_{k=1}^{n} \frac{z-a_{k}}{1-\bar{a}_{k} z}$, where $a_{k} \in D$. A infinite Blaschke product has the form $B(z)=c \prod \frac{-\bar{z}_{n}}{\left|z_{n}\right|} \frac{z-a_{n}}{1-\bar{a}_{n} z}$ where $c$ is a unimodular constant, $a_{n} \in D$ and $B(z)$ converges if and only if the zeros $a_{n}$ satisfy the condition $\sum\left(1-\left|a_{n}\right|\right)<\infty$. For some time, it was an open question whether boundary values of infinite Blaschke products are mono-components. 
Qian shows, in ([16]), that if $e^{i \theta(t)}$ is the non-tangential boundary value of an inner function, including infinite Blaschke product and singular inner function as particular cases, then the phase derivative $\theta^{\prime}(t) \geq 0$, where $\theta^{\prime}$ is defined as the non-tangential boundary value of the same quantity inside the region. The proof is a direct application of the Julia-Wolff-Caratheodory theorem in one complex variable. In other words, all phase signals are mono-components.

\section{More Nonunimodular cases}

Boundary values of starlike and $p$-starlike functions are more general monocomponents $([15],[17])$.

Definition $A$ domain $\Omega$ is said to be starlike, if $0 \in \Omega$ and $t z \in \Omega$ wherever $0<t<1$ and $z \in \Omega$. A univalent and holomorphic function $f: D \rightarrow f(D)$ is said to be starlike, if $f(D)$ is starlike and $f(0)=0$.

Closely related are convex domains and convex functions. In particular, a convex domain is a starlike domain, and a convex function is a starlike function. For a survey on starlike and convex functions in relation to mono-components, we refer to ([15]). A more general concept is $p$-starlike functions ([17]).

Definition The function $f(z)$, analytic in $D$, is said to be in the class $S(p, m)$, or $p$-starlike about zero, where $p$ and $m$ are positive integers with $p \geq m$, if and only if

(i) there exists a positive $\rho<1$ such that

$$
\operatorname{Re}\left[\frac{z f^{\prime}(z)}{f(z)}\right] \geq 0, \quad \rho<|z|<1 ;
$$

(ii)

$$
f(z)=z^{m}+a_{m+1} z^{m+1}+\cdots, \quad|z|<1 ; \text { and }
$$

$$
\int_{0}^{2 \pi} \operatorname{Re}\left[\frac{z f^{\prime}(z)}{f(z)}\right] d t=2 \pi p, \quad z=r e^{i t}, \rho<r<1
$$

(i.e., $f(z)$ is $p$-valent in $|z|<1$ ).

Möbius transform and finite Blaschke products are special cases of starlike and $p$-starlike functions. General starlike functions have both time-varying instantaneous frequencies and amplitudes. Starlike-type mono-components are more intrinsic in signal's imbedded structures.

\section{Adaptive decomposition of signals into Mono- components}

Our task is to adaptively decompose a given signal into a sum of mono-components. It seems that there are two approaches to achieve it. First, one would try to obtain a decomposition only based on the signal itself. In the second approach, 
one can seek for a large pool of mono-components and then try to decompose a signal by using the mono-components in the pool. In our practice, these two approaches are mixed together.

Theorem 5.1. ([17]) Given $f \in L^{2}(\partial D), \varepsilon_{n} \downarrow 0$, then $\exists S_{0}=c, S_{1}, \widetilde{S_{1}}, \ldots, S_{n}, \widetilde{S_{n}}$ all mono-components, such that $\left\|f-S_{0}-\sum_{k=1}^{n}\left(S_{k}+\widetilde{S_{k}}\right)\right\|_{2} \leq \varepsilon_{n}$ where the pairs $\left\{S_{k}, \widetilde{S_{k}}\right\}$ are mutually orthogonal.

As a direct consequence, we have

Corollary 5.2. Given $f \in L^{2}(\partial D), \forall \varepsilon>0$, then $\exists S_{0}, S_{1}, \widetilde{S_{1}}$, all mono-components, such that $\left\|f-S_{0}-S_{1}-\widetilde{S_{1}}\right\|_{2} \leq \varepsilon$.

The theorem and corollary show that a given signal may be decomposed into a mono-component series that converges as fast as one could desire.

The Hardy $H^{p}$ space theory supplies the following decomposition: ([6])

$$
\begin{gathered}
f \in L^{2}(\partial D) \Rightarrow f=f^{+}+f^{-} \\
f^{+} \in H^{2}(D), f^{-} \in H^{2}(\mathbb{C} \backslash \bar{D})
\end{gathered}
$$

Since $f^{+} \in H^{2}(D)$, the Nevanlinna factorization theorem guarantees that

$$
f^{+}=O_{1} I_{1}
$$

where $O_{1}$ is an outer function, and $I_{1}$ is an inner function. We further have the following programme:

$$
\begin{aligned}
f^{+} & =O_{1} I_{1} \\
& =\left(O_{1}-M_{1}\right) I_{1}+M_{1} I_{1} \\
& =z O_{2} I_{2} I_{1}+M_{1} I_{1} \\
& =z\left(O_{2}-M_{2}\right) I_{2} I_{1}+z M_{2} I_{2} I_{1}+M_{1} I_{1} \\
& =z^{2} O_{3} I_{3} I_{2} I_{1}+z M_{2} I_{2} I_{1}+M_{1} I_{1} \\
& =z^{3} O_{4} I_{4} I_{3} I_{2} I_{1}+z^{2} M_{3} I_{3} I_{2} I_{1}+z M_{2} I_{2} I_{1}+M_{1} I_{1} \\
& =\cdots
\end{aligned}
$$

where every $M_{k}$ is itself a mono-component or a sum of mono-components, $I_{k}$ are inner functions, $O_{k}$ are outer functions. Fast convergence of such decomposition is proved through comparison with the Fourier series of $f^{+}([18])$.

\section{References}

[1] E. Bedrosian, A product theorem for Hilbert transform, Proc. IEEE 51 (1963) 868-869. 
[2] B. Boashash, Estimating and interpreting the instantaneous frequency of a signal, I. Fundamentals, Proc. IEEE 80 (1992)417-430.

[3] L. Cohen, Time-Frequency Analysis, Prentice-Hall, Englewood Cliffs, NJ, 1995.

[4] Qiuhui Chen, Luoqing Li and Tao Qian, Stability of frames generated by nonlinear Fourier atoms, International Journal of Wavelets, Multiresolution and Information Processing, 3 (2005) 465-476.

[5] Qiuhui Chen, Luoqing Li and Tao Qian, Two Families of unit analytic signals with nonlinear phase, Phys. D 221 (2006) 1-12.

[6] J.B. Garnett, Bounded Analytic Functions, Academic Press, 1987.

[7] N.E. Huang, Z. Shen, S.R. Long, M.C. Wu, H.H. Shih, Q. Zheng, N.C.Yen, C. Chao Tung, H.H. Liu, The empirical mode decomposition and the Hilbert spectrum for nonlinear and nonstationary time series analysis, Proc. R. Soc. London A 454 (1998) 903-995.

[8] B. Picinbono, On instantaneous amplitude and phase of signals, IEEE Transactions on Signal Processing, 1997, 45(3): 552-560.

[9] R.C. Sharpley and V. Vatchev, Analysis of intrinsic mode functions, Constr. Approx. 24 (2006) 17-47.

[10] Tao Qian, Characterization of boundary values of functions in Hardy spaces with application in signal analysis, Journal of Integral Equations and Applications, 2005, 17(2): 159-198.

[11] Tao Qian, Qiuhui Chen, Luoqing Li, Analytic unit quadrature signals with nonlinear phase, Physica D, 2005, 203: 80-87.

[12] Tao Qian, Analytic signals and harmonic measures, Journal of Mathematical Analysis and Applications, 2006, 314: 526-536.

[13] Tao Qian, Mono-components for decomposition of signals, Mathematical Methods in the Applied Sciences (Zeitschrift fr Analysis and ihre Anwendungen), 2006, 29: 1187-1198.

[14] Tao Qian, Yuesheng Xu, Dunyan Yan, Lixin Yan, and Bo Yu, Fourier Spectrum Charaterazation Of Hardy Spaces And Applications, Proceedings Of The American Mathematical Society, Volume 137, Number 3, 971-980.

[15] Tao Qian, Mono-components for decomposition of signals, Math. Methods Appl. Sci. 29 (2006) 1187-1198.

[16] Tao Qian, Boundary Derivatives of the Phases of Inner and Outer Functions and Applications, Math. Meth. Appl. Sci. 2009, 32: 253-263 Methods in the Applied Sciences. 
[17] Tao Qian, I.T.H, I.T.Leong, and Yanbo Wang, Adaptive Decomposition of Functions Into Pieces of Non-negative Instantaneous Frequencies, accepted by International Journal of Wavelets, Multiresolution and Information Processing.

[18] Tao Qian, Lihui Tan, Yanbo Wang, Adaptive Decomposition by Weighted Inner Functions: A Generalization of Fourier Series, sumitted.

[19] Lihui Tan, Lixin Shen, and Lihua Yang, Rational orthogonal bases satisfying the Bedrosian identity, Adv Comput Math, published online: 23 June 2009.

[20] Lihui Tan, Lihua Yang, and Daren Huang, Necessary and sufficient conditions for the Bedrosian identity, J. Integral Equations Appl., Volume 21, Number1 (2009), 77-94.

[21] Yuesheng Xu and Dunyan Yan, The Bedrosian identity for the Hilbert transform of product functions, Proc. Amer. Math. Soc. 134 (2006) 2719-2728.

[22] Bo Yu and Hangzhang Zhang, The Bedrosian identity and homogeneous semi-convolution equations, J. Integral Equations Appl., Volume 20, Number4 (2008), 527-568.

[23] Lihua Yang and Haizhang Zhang, The Bedrosian identity for $H^{p}$ functions, J.Math.Anal.Appl. 345(2008) 975-984. 